\title{
Electronic and Transport Properties of Artificial Gold Chains
}

\author{
Arrigo Calzolari, ${ }^{1,2}$ Carlo Cavazzoni, ${ }^{1,3}$ and Marco Buongiorno Nardelli ${ }^{2,4}$ \\ ${ }^{1}$ INFM-S $S^{3}$ National Research Center on nanoStructures and BioSystems at Surfaces, I-41100 Modena, Italy \\ ${ }^{2}$ Department of Physics, North Carolina State University, Raleigh, North Carolina 27695, USA \\ ${ }^{3}$ CINECA, I-40033 Casalecchio di Reno, Bologna, Italy \\ ${ }^{4}$ CCS-CSM, Oak Ridge National Laboratory, Oak Ridge, Tennessee 37831, USA
}

(Received 17 May 2004; published 27 August 2004)

\begin{abstract}
We study the electronic and transport properties of artificial Au atomic chains on a NiAl(110) surface template using state-of-the-art first principles calculations. Au chains display remarkable onedimensional electronic properties that can be tuned by the selective adsorption of small molecules: a single CO group is shown to modulate the electronic wave functions, acting as a "chemical scissor" along the chain, to strongly modify the coherent transport properties of the system, and to help design one-dimensional nanodevices through artificial profiling of energy barriers.
\end{abstract}

DOI: 10.1103/PhysRevLett.93.096404

Among the revolutionary techniques brought forth by the advent of nanotechnology, the manipulation and positioning of individual atoms or molecules in precise locations in a device are both a fundamental challenge and a basic requirement for the development of nanoscale applications. The great technological strides made in the last few years have made possible the atom-by-atom fabrication of nanostructures of low dimensionality and atomic-scale dimensions. In this perspective, atomic chains (especially of $\mathrm{Au}$ and $\mathrm{Pt}$ ) that were traditionally considered toy models of little technological significance have become prototype candidates for ultimate onedimensional electronics applications.

Experimental techniques such as mechanically controllable break junctions (MCBJ) [1,2], combined with high resolution transmission electron microscopy [3] and scanning tunneling or atomic force microscopy (STM or AFM) [4] currently allow us to fabricate and analyze the structural and electronic properties of free-standing atomic chains connected to nanocontacts.

Although extremely relevant as a pioneering technique, MCBJ does not offer a definitive solution to the atomic chain fabrication problem. In fact, it is still not possible to achieve a fine control of the atomic geometry of the chain [5-7].

Recently, alternative approaches to assemble long and stable atomic chains have been proposed [8,9]. The idea is to use a supporting substrate, such as a semiconductor or metal surface, as the template for the assembling of atomic chains. Along these lines, Ho and co-workers demonstrated [9-11] that it is possible to assemble long gold chains, depositing with a STM tip single Au atoms onto a metallic $\mathrm{NiAl}(110)$ surface. The final chains are highly ordered and stable against atomic relaxation; they are frustrated to the NiAl lattice periodicity and show true metallic electronic properties in 1D. Moreover, the properties of these chains can be modified by the selective adsorption of individual molecules such as $\mathrm{CO}$, in an attempt of fine-tuning their electronic characteristics [10].
PACS numbers: 71.15.-m, 73.22.-f, 73.63.-b experimentally, there is a lack of profound theoretical understanding of the mechanisms and interactions at play in such nanostructures.

In this Letter, we have extensively investigated the properties of $\mathrm{Au}$ atomic chains on $\mathrm{NiAl}(110)$ from first principles. From the electronic point of view, our simulations, best elucidated in Fig. 1, have confirmed the remarkable 1D properties of $\mathrm{Au}$ chains on $\mathrm{NiAl}(110)$, and their independence on the local substrate. Moreover, we have shown how the selective adsorption of $\mathrm{CO}$, besides modulating the electronic wave function in the chains, acts like a "chemical scissor": it radically modifies the coherent transport properties of the system and helps in the design of one-dimensional nanodevices through artificial profiling of energy barriers.

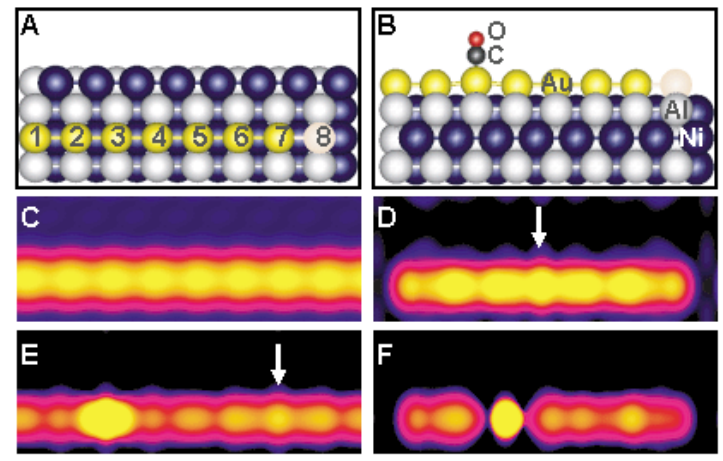

FIG. 1 (color online). (a) Top view of the gold chains on the $\mathrm{NiAl}(110)-(8 \times 2)$ surface. $($ b) Side view of the CO molecule adsorbed on $\mathrm{Au} / \mathrm{NiAl}(110)-(8 \times 2)$. Au atoms are labeled with numbers from 1 to 8 . Finite chains were simulated dropping atom $\mathrm{Au}(8)$ (light sphere) from calculations. Calculated STM images of (c) infinite Au chain; (d) finite $\mathrm{Au}_{7}$ chain; (e) CO @ infinite $\mathrm{Au}$ chain; (f) $\mathrm{CO} @$ finite $\mathrm{Au}_{7}$ chain on $\mathrm{NiAl}(110)$ surface. White arrows indicate the same symmetric STM pattern for different chains (see text). All images are calculated at $V=-0.4 \mathrm{eV}$ bias voltage. 
Our investigation is based on a recently proposed approach to calculating both the electronic and the coherent transport properties of extended nanostructures from first principles [12], where the ground state electronic structure is obtained through a state-of-the-art ab initio methodology based on the density functional theory (DFT) and a plane wave representation [13]. The coherent transport properties have been computed using the WANT code [14], which uses a Green's function formalism based on "maximally localized Wannier functions" (MLWF's) [15] as a minimal basis set [12].

As a first step in our investigation, we have studied the structural and electronic properties of the chains obtained from the controlled deposition of $\mathrm{Au}$ atoms on the clean $\mathrm{NiAl}(110)$ surface. The NiAl (110) surface consists of alternating rows of $\mathrm{Ni}$ and $\mathrm{Al}$ separated by $2.9 \AA$ $(4.2 \AA)$ along the $[001]([1 \overline{1} 0])$ direction. The optimized structure exhibits a vertical relaxation of the first layer with a $0.2 \AA$ relative displacement between the outward $\mathrm{Al}$ and the inward $\mathrm{Ni}$ atoms, in agreement with previous calculations [16]. The surface is metallic and nonmagnetic, with a net charge accumulation around the $\mathrm{Ni}$ atoms, which, together with the ripple relaxation, offers a natural template for the deposition of the Au chain.

$\mathrm{Au}$ atoms deposited on bridge positions in the $\mathrm{Ni}$ troughs along the [001] direction form monatomic chains as seen in experiments [9] and shown in Fig. 1(a). The relaxed wires are thermodynamically stable and do not present structural distortions: the presence of the ordered substrate drives the formation of an ordered Au wire with an interatomic distance of $2.9 \AA$, as induced by the Ni-Ni periodicity, independently of the length of the chain. The interatomic separation is shorter than that observed in typical MCBJ experiments under stretching [1-3], but larger than the DFT equilibrium distance in isolated chains [5-7]. A careful analysis of the electronic structure of the $\mathrm{Au} / \mathrm{NiAl}$ interface shows that the formation of the gold chain occurs without a measurable modification of the substrate, which acts solely as an inert template for the formation of the wire. Because of the low coordination number, the $6 s$ valence electrons of $\mathrm{Au}$, slightly hybridized with the inner $5 d$ shell, lose their spherical symmetry and overlap along the chain, giving rise to highly directional $\sigma$ bonds between neighboring sites. In the infinite chain these $\sigma$ states form a metallic band, superimposed - with negligible modification - to the 2D band structure of the clean $\mathrm{NiAl}(110)$ surface. The Au band, across the Fermi level (FL), lies in an internal lens of the surface band structure [16]: the absence of surface states in that energy region supports the preference of Au to interact with neighboring adatoms instead of with the substrate. Indeed, the reciprocal interactions reduce to a mutual screening at the interface, without sharing of charge. Justified by the above observations, we have analyzed the nature of the bonding in the $\mathrm{Au}$ chain computing the MLWF's for an isolated wire, constraining the atoms to the adsorbate configuration. The results are shown in Fig. 2(a) where we reproduce the same $\sigma$ bonding orbital as previously described.

To prove further the correctness of this interpretation, we have also studied the transport properties [12] of such chains. Our results are reported in Fig. 3. The dashed (red) line describes the bulk [12] transmittance through the gold chain adsorbed on the surface. The curve has been obtained directly from the $\mathrm{Au} / \mathrm{NiAl}(110)$ calculation, exploiting the localization properties of the Wannier functions, to extract the contributions of the Au chain from the total transmittance of the system. The shaded area, instead, is the bulk transmittance of the isolated periodic chain (without the substrate). In both cases, for a large part of the spectrum, the transmittance is dominated by a single-channel deriving from the $\sigma$ states [see Fig. 2(a)], while the $5 d$ states of Au contribute only in the energy range $\sim(-2,-0.5) \mathrm{eV}$ below the Fermi level. The almost complete overlap of the two curves confirms that, even in the presence of the substrate, the gold chain behaves, indeed, as an isolated system.

Turning to the characterization of the electronic properties, in Fig. 1(c) we present the calculated STM image [17] at constant current and bias voltage $-0.4 \mathrm{eV}$, for the $\mathrm{Au} / \mathrm{NiAl}(110)$ system. The image confirms the intrinsic 1D nature of the chain: the bright protrusions of the plot correspond to the $\sigma$ band across the Au chain, while the $\mathrm{Au} 5 d$ states and the substrate, lying at much lower energies, do not contribute to the STM image.

To get closer to the experimental configurations [9], we have simulated a finite chain of gold (labeled $\mathrm{Au}_{7}$ ) on a $\mathrm{NiAl}(110)-(8 \times 2)$ surface, dropping one atom (light sphere in Fig. 1(a)] off the periodic chain. The relaxed structure does not show structural modifications, but a slight atomic distortion due to the frustration of the $\sigma$ bond at the boundaries of the chain. Passing from infinite to finite wire, however, the delocalized Bloch-like states transform into molecular orbitals that are symmetrically distributed around the center of the chain [atom $\mathrm{Au}(4)$ ] as shown in Fig. 1(d). The different intensities of the STM spots are the manifestation of the resonance effects on the wave functions, due to the finite size of the $\mathrm{Au}_{7}$ wire. Our

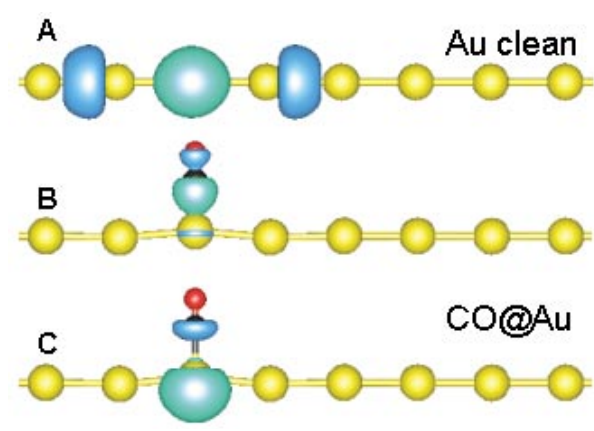

FIG. 2 (color online). Isosurface of selected Wannier functions centered on the $\mathrm{Au}^{(3)}$ atom in clean (a), and $\mathrm{CO} @ \mathrm{Au}$ chains (b),(c). (a) Au-Au $\sigma$ bonding; (b) $\mathrm{Au} \leftarrow \mathrm{C} \sigma$ bonding; (c) unpaired $\mathrm{Au}_{6 s}$-derived state. 


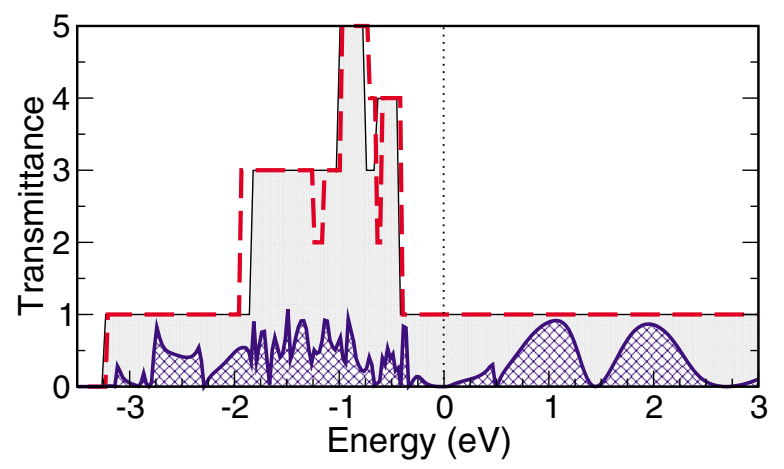

FIG. 3 (color online). Transmittance of Au chain in the isolated configuration (shaded area), in the $\mathrm{Au} / \mathrm{NiAl}$ interface (dashed red line), and after $\mathrm{CO}$ adsorption (squared blue area). Vertical dotted line represents the Fermi level.

simulated image accurately reproduces the experimental topographic results [9] and confirms once again that the $\mathrm{Au} / \mathrm{NiAl}(110)$ composite represents a prototype system, where the surface behaves as a structural template for the formation of the artificial one-dimensional wire.

The controlled formation of Au atomic wires allows us to further investigate possible techniques to manipulate, in situ, the electronic properties of such nanostructures. Following Ref. [12], we have studied the adsorption of a carbon monoxide molecule on top of a selected atom [labeled $\mathrm{Au}(3)]$ of the chain on the $\mathrm{NiAl}(110)-8 \times 2$ template, as shown in Fig. 1(b), both for the infinite (periodic) and finite $\mathrm{Au}_{7}$ wire. In both cases the adsorption is hexotermic, with an energy gain of 0.41 and $0.26 \mathrm{eV} /$ cell, respectively. The adsorption of CO locally distorts the chain: $\mathrm{Au}(3)$ slightly moves vertically out of the surface $(\Delta z=0.2 \AA)$, to bind to the CO molecule at a distance $d[\mathrm{Au}(3)-C]=1.96 \AA$. Neighboring atoms do not undergo further changes. In the case of the finite $\mathrm{Au}_{7}$ chain, we observe a minor atomic distortion at the ends, due to boundary effects. As before, the substrate does not directly couple with the Au adatoms.

Despite the small structural modifications, the adsorption of carbon monoxide drastically changes the electronic properties of the system, acting as a chemical scissor, in agreement with the experimental findings [10]. The molecule selectively cuts the chain in two parts that become electronically separated. The STM images for periodic and finite chains [Figs. 1(e) and 1(f)] show that $\mathrm{CO}$ pins the electronic states of the corresponding $\mathrm{Au}$ atom, interrupting the supramolecular delocalization of the chain orbitals. In the periodic wire [Fig. 1(e)], the CO adsorption induces the formation of an effective sevenatom-long chain separated by the Au-CO (brightest spot in the plot) complex: it is, in fact, possible to recognize the symmetric STM pattern, which characterizes the finite $\mathrm{Au}_{7}$ chain (see white arrows in Figs. 1(d) and 1(e)]. The cutting effect is even stronger in the short chain [Figs. 1(f)], where the formation of a dimer and a tetramer is evident. This feature is confirmed by the comparison with DFT results about isolated Au dimers [18].

The electronic and chemical properties of the Au-CO complex can be best understood via the analysis of the individual bonding orbitals as represented by the MLWF's. Figure 2(a) displays the Au-Au bonding state that characterizes the clean Au wire. Similar MLWF's are observed also in the presence of $\mathrm{CO}$, except for the $\mathrm{Au}(3)$ site. This evidence confirms that the carbon monoxide modifies the bonding pattern of the adsorption site only, where we observe clear electronic signatures of the formation of the Au-CO complex. It is well known that, although carbon is less electronegative than oxygen, in the $\mathrm{CO}$ molecule $\mathrm{C}$ bears a formal negative charge $(-1)$ [19], as best represented in the Lewis structure for carbon monoxide : $\mathrm{C} \equiv \mathrm{O}$ : (where a dot stands for a singleelectron and a hyphen for two electrons in a bond). In order to saturate this electron concentration, in the metalcarbonyl compound $\mathrm{CO}$ tends to act as donor ligand towards the transition metal ( $\mathrm{Au}-\mathrm{C} \equiv \mathrm{O}:$ ). The formation of the $\mathrm{Au} \leftarrow \mathrm{C} \sigma$ bond involves the unshared electron pair of $\mathrm{C}$ and the empty $6 p_{z}$ orbital of $\mathrm{Au}$ [19], but not its $6 s$ valence electron, which remains, in principle, available for further bonding. This chemical mechanism is confirmed by the analysis of the MLWF's for these bonding states. Figure 2(b) shows the Au-CO $\sigma$ bond, which derives from the lone pair of the isolated molecule, while the electronic state of the unpaired $6 s$ electron is reported in Fig. 2(c). Two facts are worthy of note: first, the repulsive interaction induced by the $\mathrm{Au}-\mathrm{C}$ bond on site $\mathrm{Au}(3)$ pushes the $6 s$ level towards a higher energy, hindering the formation of bonds with neighboring atoms along the chain. Second, the $6 s$ unpaired state shows a strong polarized character along the direction parallel to $\mathrm{CO}$ and perpendicular to the chain. Thus, although globally the system remains charge neutral, the $\mathrm{Au}(3)$ atom results are formally charged, because of its $6 s$ electron. The presence of such an unpaired electron justifies both the electronic breaking of the chain and the fact that the brightest spots on the STM images of Figs. 1(e) and 1(f) are, indeed, observed on $\mathrm{Au}(3)$.

The localization of electronic charge induced by the $\mathrm{CO}$ adsorption drastically affects the transport properties of the whole chain, as reported in Fig. 3. Here, the squared (blue) area is the transmittance of an open device where clean semi-infinite Au wires constitute the leads and the CO@Au act as the conductor [12]. In comparison with the clean system (shaded gray area), we see a dramatic reduction of the transmittance in the whole energy range, caused by the misalignment of electronic states between the conductor and the leads. Indeed, the CO adsorption induces a metal-insulator transition in the Au wire, opening a gap at the Fermi level. The sharp modification of quantum conductance (transmittance at FL) from 1 to $0 e^{2} / h$ is the signature of the chemical scissor effect of $\mathrm{CO}$ on $\mathrm{Au}$ nanowires and suggests possible nanotechnology applications, such as quantum

096404-3 


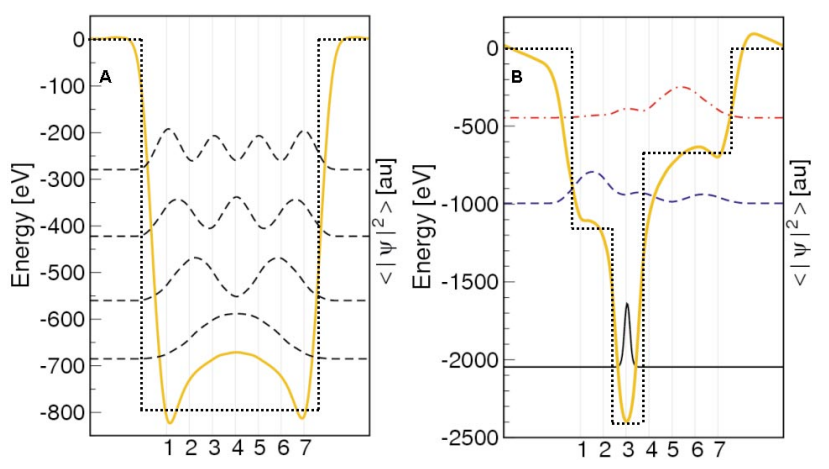

FIG. 4 (color online). Average DFT (straight line) and steplike model (dotted line) of total potentials for (a) clean $\mathrm{Au}_{7}$ and (b) $\mathrm{CO} @ \mathrm{Au}_{7}$ systems. Average square modulus of lowest energies DFT eigenfunctions (dash-dotted line) are shown; the energy separations are arbitrary. Vertical lines indicate the positions of $\mathrm{Au}$ atoms (1-7) along the chains.

switches controlled at the atomic level, or nanoleads for molecular electronics. The latter hypothesis, experimentally advanced in the case of a $\mathrm{Cu}(\mathrm{II})$-phthalocyanine bonded to two Au chains [11], suggests the possibility of manufacturing prototypical nanojunctions, where the leads are not undefined electron reservoirs, but controlled channels for electron transport. In this case, the flow or the suppression of the current should be directly related to the formation or the breaking of chemical bonds between the Au chain and site-specific molecules.

This idea of exploiting the Au chains as single-channel systems also suggests the possibility of profiling energy barriers in 1D nanostructures, to achieve a full control on the engineering of artificial 1D electronic devices. All the electronic characteristics of these systems can be described using a simple "particle-in-a-box" model, where the boundary of finite chains and the adsorption of external molecules constitute local constraints for the wave functions [9].

Figure 4 shows the total potential seen by an electron averaged perpendicular to the chain direction (straight line) for (a) a clean $\mathrm{Au}_{7}$, and (b) a $\mathrm{CO} @ \mathrm{Au}_{7}$ chain. In this same figure we have superimposed (dashed lines) the average square moduli of the DFT eigenfunctions corresponding to the lowest energy eigenvalues. As expected for a particle-in-a-box system, in the case of clean chain [Fig. 4(a)], the ab initio wave functions have the character of those of a deep $(\sim \infty)$ square potential well. We, indeed, proved this feature numerically via the solution of the Schrödinger equation for a toy potential profile (dotted line in Fig. 4) design to fit the DFT one. Assuming $V=-780 \mathrm{eV}$ (depth) and $L=20.3 \AA$ (the $\mathrm{Au}_{7}$ length), we obtained the same spectrum (within $\pm 40 \mathrm{meV}$ ) for the lowest energy eigenvalues. In the case of the $\mathrm{CO} @ \mathrm{Au}_{7}$ chains, the potential profile may by separated into three parts: a dimer (positions 1,2), a monomer (3), and a tetramer $(4,7)$. The shorter the segment is, the deeper the well due to the increased localization is. In particular, the lowest energy wave functions are localized around the $\mathrm{Au}-\mathrm{CO}$ site (black thin line), while those at higher energy are mainly localized in one of the two Au segments.

These results demonstrate that in the 1D chain we can engineer an arbitrary potential profile where electrons behave as particles-in-a-box making use of the selective adsorption and atom-by-atom manipulation of the structure of the system. It is then not difficult to envisage the design of 1D devices with chosen profiles where singleelectron tunneling can be tuned at will, greatly enhancing the promise of these systems for nanotechnological applications and single-electron electronics.

We thank A. Catellani and M. Rontani for invaluable help and illuminating discussions. This work was supported in part by MIUR (Italy) through Grant FIRBNomade and by INFM through "Progetto calcolo parallelo," the Petroleum Research Fund of the ACS, the Mathematical Information and Computer Science Division, Office of Advanced Scientific Computing Research of the U.S. Department of Energy under Contract No. DE-AC05-00OR22725 with UT-Battelle and DOE-SC Grant "Integrated Multiscale Modeling of Molecular Computing Devices."

[1] A. I. Yanson et al., Nature (London) 395, 783 (1998).

[2] R. H. M. Smit et al., Nature (London) 419, 906 (2002).

[3] H. Ohnishi, Y. Kondo, and K. Takayanagi, Nature (London) 395, 780 (1998).

[4] G. Rubio-Bollinger et al., Phys. Rev. Lett. 87, 026101 (2001).

[5] D. Sanchez-Portal et al., Phys. Rev. Lett. 83, 3884 (1999).

[6] S. R. Bahn and K.W. Jacobsen, Phys. Rev. Lett. 87, 266101 (2001).

[7] S. B. Legoas et al., Phys. Rev. Lett. 88, 076105 (2002).

[8] J. N. Crain et al., Phys. Rev. Lett. 90, 176805 (2003); S. C. Erwin, Phys. Rev. Lett. 91, 206101 (2003).

[9] N. Nilius, T. M. Wallis, and W. Ho, Science 297, 1853 (2002).

[10] N. Nilius, T. M. Wallis, and W. Ho, Phys. Rev. Lett. 90, 186102 (2003).

[11] G.V. Nazin, X. H. Qiu, and W. Ho, Science 302, 77 (2003).

[12] A. Calzolari et al., Phys. Rev. B 69, 035108 (2004).

[13] Calculations in this work have been done using the PWSCF package (S. Baroni et al., www.pwscf.org).

[14] A. Calzolari et al., WANT code, www.wanniertransport.org. See also Ref. [12].

[15] N. Marzari and D. Vanderbilt, Phys. Rev. B 56, 12847 (1997).

[16] S. C. Lui et al., Phys. Rev. B 39, 13149 (1989).

[17] J. Tersoff and D. R. Hamman, Phys. Rev. B 31, 805 (1985).

[18] N. Nilius et al., Phys. Rev. Lett. 90, 196103 (2003).

[19] F. A. Cotton et al., Advanced Inorganic Chemistry (Wiley \& Sons, Inc., New York, 1999). 